\title{
New generalized fractional versions of Hadamard and Fejér inequalities for harmonically convex functions
}

\author{
Xiaoli Qiang ${ }^{1}$, Ghulam Farid ${ }^{2 *}$, Muhammad Yussouf ${ }^{3 \dagger}$, Khuram Ali Khan $^{3 \dagger}$ and Atiq Ur Rahman ${ }^{2 \dagger}$
}

"Correspondence:

faridphdsms@hotmail.com ${ }^{2}$ Department of Mathematics, COMSATS University Islamabad, Attock Campus, Attock, Pakistan Full list of author information is available at the end of the article ${ }^{\dagger}$ Equal contributors

\begin{abstract}
The aim of this paper is to establish new generalized fractional versions of the Hadamard and the Fejér-Hadamard integral inequalities for harmonically convex functions. Fractional integral operators involving an extended generalized Mittag-Leffler function which are further generalized via a monotone increasing function are utilized to get these generalized fractional versions. The results of this paper give several consequent fractional inequalities for harmonically convex functions for known fractional integral operators deducible from utilized generalized fractional integral operators.
\end{abstract}

Keywords: Harmonically convex function; Hadamard inequality; Fejér-Hadamard inequality; Mittag-Leffler function; Fractional integral operators

\section{Introduction}

Fractional integral inequalities are generalizations of classical integral inequalities. Hadamard and Fejér-Hadamard inequalities are the inequalities which have been studied extensively for different fractional integral/derivative operators, see [1, 4-6, 8$10,14,16,17,23,25,27,30,33,34,36-38,42,44]$. The main objective of this paper is to prove some new fractional generalizations of Hadamard and the Fejér-Hadamard inequalities for harmonically convex functions. We begin with fractional integral operators defined by Salim and Faraj in [35] containing generalized Mittag-Leffler function in their kernels as follows:

Definition 1 ([35]) Let $\sigma, \tau, k, r, \rho$ be positive real numbers and $\omega \in \mathbb{R}$. Then the generalized fractional integral operators containing Mittag-Leffler function for a real-valued continuous function $f$ are defined by

$$
\begin{aligned}
& \left(\epsilon_{\sigma, \tau, \delta, \omega, a}^{\rho, r, k} f\right)(x)=\int_{a}^{x}(x-t)^{\tau-1} E_{\sigma, \tau, \delta}^{\rho, r, k}\left(\omega(x-t)^{\sigma}\right) f(t) d t, \\
& \left(\epsilon_{\sigma, \tau, \delta, \omega, b}^{\rho, r, k} f\right)(x)=\int_{x}^{b}(t-x)^{\tau-1} E_{\sigma, \tau, \delta}^{\rho, r, k}\left(\omega(t-x)^{\sigma}\right) f(t) d t,
\end{aligned}
$$

(c) The Author(s) 2020. This article is licensed under a Creative Commons Attribution 4.0 International License, which permits use, sharing, adaptation, distribution and reproduction in any medium or format, as long as you give appropriate credit to the original author(s) and the source, provide a link to the Creative Commons licence, and indicate if changes were made. The images or other third party material in this article are included in the article's Creative Commons licence, unless indicated otherwise in a credit line to the material. If material is not included in the article's Creative Commons licence and your intended use is not permitted by statutory regulation or exceeds the permitted use, you will need to obtain permission directly from the copyright holder. To view a copy of this licence, visit http://creativecommons.org/licenses/by/4.0/. 
where $E_{\sigma, \tau, \delta}^{\rho, r, k}(t)$ is the generalized Mittag-Leffler function defined as

$$
E_{\sigma, \tau, \delta}^{\rho, r, k}(t)=\sum_{n=0}^{\infty} \frac{(\rho)_{k n} t^{n}}{\Gamma(\sigma n+\tau)(r)_{\delta n}} .
$$

The connection of Mittag-Leffler function with fractional calculus is very useful and well-established. Its alliance with fractional integral operators as a kernel plays a vital role in the development of the theory and applications of fractional calculus in various subjects of science and engineering [12, 13, 18-22, 24, 28, 29, 31, 35, 39, 43]. In [2] Andrić et al. defined the following fractional integral operators containing an extended generalized Mittag-Leffler function in their kernels:

Definition $2([2])$ Let $\omega, \tau, \delta, \rho, c \in \mathbb{C}, \mathfrak{R}(\tau), \mathfrak{R}(\delta)>0, \mathfrak{R}(c)>\mathfrak{R}(\rho)>0$, with $p \geq 0, \sigma, r>0$ and $0<k \leq r+\sigma$. Let $f \in L_{1}[a, b]$ and $x \in[a, b]$. Then the generalized fractional integral operators $\epsilon_{\sigma, \tau, \delta, \omega, a^{+}}^{\rho, r, k, c} f$ and $\epsilon_{\sigma, \tau, \delta, \omega, b^{-}}^{\rho, r, k, c} f$ are defined by

$$
\begin{aligned}
& \left(\epsilon_{\sigma, \tau, \delta, \omega, a^{+}}^{\rho, r, k, c} f\right)(x ; p)=\int_{a}^{x}(x-t)^{\tau-1} E_{\sigma, \tau, \delta}^{\rho, r, k, c}\left(\omega(x-t)^{\sigma} ; p\right) f(t) d t, \\
& \left(\epsilon_{\sigma, \tau, \delta, \omega, b^{-}}^{\rho, r, k, c} f\right)(x ; p)=\int_{x}^{b}(t-x)^{\tau-1} E_{\sigma, \tau, \delta}^{\rho, r, k, c}\left(\omega(t-x)^{\sigma} ; p\right) f(t) d t,
\end{aligned}
$$

where

$$
E_{\sigma, \tau, \delta}^{\rho, r, k, c}(t ; p)=\sum_{n=0}^{\infty} \frac{\beta_{p}(\rho+n k, c-\rho)(c)_{n k} t^{n}}{\beta(\rho, c-\rho) \Gamma(\sigma n+\tau)(\delta)_{n r}}
$$

is the extended generalized Mittag-Leffler function.

In [7] (see, also [26]) Farid elegantly defined a unified integral operator as follows:

Definition 3 Let $f, g:[a, b] \rightarrow \mathbb{R}, 0<a<b$ be functions such that $f$ is positive, $f \in L_{1}[a, b]$, and $g$ is differentiable and strictly increasing. Also let $\frac{\phi}{x}$ be an increasing function on $[a, \infty)$ and $\omega, \tau, \delta, \rho, c \in \mathbb{C}, \mathfrak{N}(\tau), \mathfrak{R}(\delta)>0, \mathfrak{R}(c)>\mathfrak{N}(\rho)>0$, with $p \geq 0, \sigma, r>0$ and $0<k \leq r+\sigma$. Then for $x \in[a, b]$ the integral operators $\left({ }_{g} F_{\sigma, \tau, \delta, \omega, a^{+}}^{\phi, \rho, r, k, c} f\right)$ and $\left({ }_{g} F_{\sigma, \tau, \delta, \omega, b^{-}}^{\phi, \rho, r, k, c} f\right)$ are defined by

$$
\begin{aligned}
& \left({ }_{g} F_{\sigma, \tau, \delta, \omega, a^{+}}^{\phi, \rho, r, k} f\right)(x ; p)=\int_{a}^{x} \frac{\phi(g(x)-g(t))}{g(x)-g(t)} E_{\sigma, \tau, \delta}^{\rho, r, k, c}\left(\omega(g(x)-g(t))^{\sigma} ; p\right) f(t) d(g(t)), \\
& \left({ }_{g} F_{\sigma, \tau, \delta, \omega, b^{-}}^{\phi, \rho, r, k, c} f\right)(x ; p)=\int_{x}^{b} \frac{\phi(g(t)-g(x))}{g(t)-g(x)} E_{\sigma, \tau, \delta}^{\rho, r, k, c}\left(\omega(g(t)-g(x))^{\sigma} ; p\right) f(t) d(g(t)) .
\end{aligned}
$$

A generalization of integral operators defined in (1.4), (1.5) can be deduced from the above definition by taking $\phi(t)=t^{\tau}$ as follows:

Definition 4 Let $f, g:[a, b] \rightarrow \mathbb{R}, 0<a<b$ be functions such that $f$ is positive, $f \in L_{1}[a, b]$, and $g$ is differentiable and strictly increasing. Also let $\omega, \tau, \delta, \rho, c \in \mathbb{C}, \Re(\tau), \Re(\delta)>0, \Re(c)>$ $\mathfrak{R}(\rho)>0$, with $p \geq 0, \sigma, r>0$, and $0<k \leq r+\sigma$. Then for $x \in[a, b]$ the integral operators 
are defined by

$$
\begin{aligned}
& \left({ }_{g} \Upsilon_{\sigma, \tau, \delta, \omega, a^{+}}^{\rho, r, k, c} f\right)(x ; p)=\int_{a}^{x}(g(x)-g(t))^{\tau-1} E_{\sigma, \tau, \delta}^{\rho, r, k, c}\left(\omega(g(x)-g(t))^{\sigma} ; p\right) f(t) d(g(t)), \\
& \left({ }_{g} \Upsilon_{\sigma, \tau, \delta, \omega, b^{-}}^{\rho, r, k, c} f\right)(x ; p)=\int_{x}^{b}(g(t)-g(x))^{\tau-1} E_{\sigma, \tau, \delta}^{\rho, r, k, c}\left(\omega(g(t)-g(x))^{\sigma} ; p\right) f(t) d(g(t)) .
\end{aligned}
$$

Fractional integral operators (1.9), (1.10) produce some already known integral operators, see [33, Remark 1].

We are interested in utilizing fractional integral operators (1.9), (1.10) for the establishment of Hadamard and Fejér-Hadamard inequalities for harmonically convex functions. The classical Hadamard inequality is an elegant geometric interpretation of convex functions.

Definition 5 ([41]) A function $f:[a, b] \rightarrow \mathbb{R}$ is said to be convex if

$$
f(t x+(1-t) y) \leq t f(x)+(1-t) f(y)
$$

holds for all $x, y \in[a, b]$ and $t \in[0,1]$.

Hadamard inequality is stated in the following theorem:

Theorem 1.1 Let $f:[a, b] \rightarrow \mathbb{R}, a<b$, be a convex function. Then the following inequality holds:

$$
f\left(\frac{a+b}{2}\right) \leq \frac{1}{b-a} \int_{a}^{b} f(x) d x \leq \frac{f(a)+f(b)}{2} .
$$

Fejér-Hadamard inequality is a weighted version of Hadamard inequality proved by $\mathrm{Fe}-$ jér in [11] which is stated in the following theorem:

Theorem 1.2 Let $f:[a, b] \rightarrow \mathbb{R}$ be a convex function and $g:[a, b] \rightarrow \mathbb{R}$ be a nonnegative, integrable, and symmetric about $\frac{a+b}{2}$. Then the following inequality holds:

$$
f\left(\frac{a+b}{2}\right) \int_{a}^{b} g(x) d x \leq \int_{a}^{b} f(x) g(x) d x \leq \frac{f(a)+f(b)}{2} \int_{a}^{b} g(x) d x .
$$

Next we give the definition of harmonically convex functions [14].

Definition 6 Let $I$ be an interval of nonzero real numbers. Then a function $f: I \rightarrow \mathbb{R}$ is said to be harmonically convex if

$$
f\left(\frac{a b}{t a+(1-t) b}\right) \leq t f(b)+(1-t) f(a)
$$

holds for all $a, b \in I$ and $t \in[0,1]$. If the reversed inequality holds in (1.13), then $f$ is called a harmonically concave function.

Example $1.3([14])$ Let $f:(0, \infty) \rightarrow \mathbb{R}, f(x)=x$, and $g:(-\infty, 0) \rightarrow \mathbb{R}, g(x)=x$. Then $f$ is a harmonically convex function and $g$ is a harmonically concave function. 
The above example gives following result.

Proposition 1.4 ([14]) Let $I \subset \mathbb{R} \backslash\{0\}$ be a real interval and $f: I \rightarrow \mathbb{R}$ is a function.

(i) If $I \subset(0, \infty)$ and $f$ is convex and nondecreasing function, then is harmonically convex.

(ii) If $I \subset(0, \infty)$ and $f$ is harmonically convex and nonincreasing function, then $f$ is convex.

(iii) If $I \subset(-\infty, 0)$ and $f$ is harmonically convex and nondecreasing function, then $f$ is convex.

(iv) If $I \subset(-\infty, 0)$ and $f$ is convex and nonincreasing function, then $f$ is a harmonically convex.

Definition 7 ([25]) A function $\varphi:[a, b] \subset \mathbb{R} \backslash\{0\} \rightarrow \mathbb{R}$ is said to be harmonically symmetric about $\frac{a+b}{2 a b}$ if

$$
\varphi\left(\frac{1}{x}\right)=\varphi\left(\frac{1}{\frac{1}{a}+\frac{1}{b}-x}\right), \quad x \in[a, b]
$$

For some recent work on harmonically convex functions, we refer readers to $[1,3,9,14$, $25,30]$ and references therein. In this paper, we extend the work of Abbas et al. [1] and Farid et al. [9] for Hadamard and Fejér-Hadamard-type inequalities by using (1.9) and (1.10).

In Sect. 3, we prove two fractional versions of Hadamard and two fractional versions of Fejér-Hadamard-type inequalities for harmonically convex functions by using fractional integral operators (1.9) and (1.10). Furthermore, the associated published results are obtained which are identified in remarks, some corollaries are also given.

\section{Main results}

Theorem 2.1 Let $f, g:[a, b] \rightarrow \mathbb{R}, 0<a<b$, Range $(g) \subset[a, b]$, be functions such that $f$ is positive, $f \in L_{1}[a, b]$, and $g$ is differentiable and strictly increasing. Iff is a harmonically convex function on $[a, b]$, then for fractional integral operators (1.9) and (1.10) we have

$$
\begin{aligned}
& f\left(\frac{2 g(a) g(b)}{g(a)+g(b)}\right)\left({ }_{g} \Upsilon_{\sigma, \tau, \delta, \omega^{\prime},\left(g^{-1}\left(\frac{1}{g(a)}\right)\right)}^{\rho, r, k)}-1\right)\left(g^{-1}\left(\frac{1}{g(b)}\right) ; p\right) \\
& \leq \frac{1}{2}\left(\left({ }_{g} \Upsilon_{\sigma, \tau, \delta, \omega^{\prime},\left(g^{-1}\left(\frac{1}{g(a)}\right)\right)}^{\rho, r, k} f \circ \psi\right)\left(g^{-1}\left(\frac{1}{g(b)}\right) ; p\right)\right. \\
& \left.+\left({ }_{g} \Upsilon_{\sigma, \tau, \delta, \omega^{\prime},\left(g^{-1}\left(\frac{1}{g(b)}\right)\right)}^{\rho, r, k} f \circ \psi\right)\left(g^{-1}\left(\frac{1}{g(a)}\right) ; p\right)\right) \\
& \leq \frac{f(g(a))+f(g(b))}{2}\left({ }_{g} \Upsilon_{\sigma, \tau, \delta, \omega^{\prime},\left(g^{-1}\left(\frac{1}{g(b)}\right)\right)^{+}}^{\rho, r, k)}\left(g^{-1}\left(\frac{1}{g(a)}\right) ; p\right),\right.
\end{aligned}
$$

where $\psi(t)=\frac{1}{g(t)}$ for all $t \in\left[\frac{1}{b}, \frac{1}{a}\right]$ and $\omega^{\prime}=\omega\left(\frac{g(a)(b)}{g(b)-g(a)}\right)^{\sigma}$.

Proof Since $f$ is harmonically convex on $[a, b]$, for $x, y \in[a, b]$, the following inequality holds:

$$
f\left(\frac{2 g(x) g(y)}{g(x)+g(y)}\right) \leq \frac{f(g(x))+f(g(y))}{2} .
$$


By taking $g(x)=\frac{g(a) g(b)}{\operatorname{tg}(b)+(1-t) g(a)}$ and $g(y)=\frac{g(a) g(b)}{\operatorname{tg}(a)+(1-t) g(b)}$ in (2.2), we have

$$
2 f\left(\frac{2 g(a) g(b)}{g(a)+g(b)}\right) \leq f\left(\frac{g(a) g(b)}{\operatorname{tg}(b)+(1-t) g(a)}\right)+f\left(\frac{g(a) g(b)}{\operatorname{tg}(a)+(1-t) g(b)}\right) .
$$

Multiplying (2.3) by $t^{\tau-1} E_{\sigma, \tau, \delta}^{\rho, r, k, c}\left(\omega\left(t^{\sigma}\right) ; p\right)$ and integrating over [0,1], we get

$$
\begin{aligned}
2 f( & \left.\frac{2 g(a) g(b)}{g(a)+g(b)}\right) \int_{0}^{1} t^{\tau-1} E_{\sigma, \tau, \delta}^{\rho, r, k, c}\left(\omega t^{\sigma} ; p\right) d t \\
\leq & \int_{0}^{1} t^{\tau-1} E_{\sigma, \tau, \delta}^{\rho, r, k, c}\left(\omega t^{\sigma} ; p\right) f\left(\frac{g(a) g(b)}{\operatorname{tg}(b)+(1-t) g(a)}\right) d t \\
& \quad+\int_{0}^{1} t^{\tau-1} E_{\sigma, \tau, \delta}^{\rho, r, k, c}\left(\omega t^{\sigma} ; p\right) f\left(\frac{g(a) g(b)}{\operatorname{tg}(a)+(1-t) g(b)}\right) d t .
\end{aligned}
$$

By setting $g(x)=\frac{\operatorname{tg}(b)+(1-t) g(a)}{g(a) g(b)}$ and $g(y)=\frac{\operatorname{tg}(a)+(1-t) g(b)}{g(a) g(b)}$ in (2.4) and using (1.9), (1.10), the first inequality of (2.1) can be obtained. On the other hand, using harmonic convexity of $f$, we have

$$
f\left(\frac{g(a) g(b)}{t g(b)+(1-t) g(a)}\right)+f\left(\frac{g(a) g(b)}{\operatorname{tg}(a)+(1-t) g(b)}\right) \leq f(g(a))+f(g(b)) .
$$

Multiplying (2.5) by $t^{\tau-1} E_{\sigma, \tau, \delta}^{\rho, r, k, c}\left(\omega\left(t^{\sigma}\right) ; p\right)$ and then integrating over $[0,1]$, we get

$$
\begin{aligned}
& \int_{0}^{1} t^{\tau-1} E_{\sigma, \tau, \delta}^{\rho, r, k, c}\left(\omega t^{\sigma} ; p\right) f\left(\frac{g(a) g(b)}{\operatorname{tg}(b)+(1-t) g(a)}\right) d t \\
& \quad+\int_{0}^{1} t^{\tau-1} E_{\sigma, \tau, \delta}^{\rho, r, k, c}\left(\omega t^{\sigma} ; p\right) f\left(\frac{g(a) g(b)}{\operatorname{tg}(a)+(1-t) g(b)}\right) d t \\
& \leq(f(g(a))+f(g(b))) \int_{0}^{1} t^{\tau-1} E_{\sigma, \tau, \delta}^{\rho, r, k}\left(\omega t^{\sigma} ; p\right) d t .
\end{aligned}
$$

By setting $g(x)=\frac{\operatorname{tg}(b)+(1-t) g(a)}{g(a) g(b)}$ and $g(y)=\frac{\operatorname{tg}(a)+(1-t) g(b)}{g(a) g(b)}$ in (2.6), and using (1.9), (1.10), the second inequality of (2.1) can be obtained.

\section{Remark 2.2}

(i) By setting $p=0$ and $g=I$, [1, Theorem 3.1] is obtained.

(ii) By setting $g=I$, [9, Theorem 2.1] is obtained.

(iii) By setting $\omega=p=0, g=I,[15$, Theorem 4$]$ is obtained.

Corollary 2.3 If we take $\psi(x)=x$ in Theorem 2.1, then we get the following inequalities:

$$
\begin{aligned}
& f\left(\frac{2}{a+b}\right)\left({ }_{g} \Upsilon_{\sigma, \tau, \delta, \omega^{\prime},\left(\frac{1}{a}\right)^{-}}^{\rho, r, k)} 1\right)\left(\frac{1}{b} ; p\right) \\
& \quad \leq \frac{1}{2}\left(\left({ }_{g} \Upsilon_{\sigma, \tau, \delta, \omega^{\prime},\left(\frac{1}{a}\right)^{-}}^{\rho, r, k, c} f\right)\left(\frac{1}{b} ; p\right)+\left({ }_{g} \Upsilon_{\sigma, \tau, \delta, \omega^{\prime},\left(\frac{1}{b}\right)^{+}}^{\rho, r, k)}\right)\left(\frac{1}{a} ; p\right)\right) \\
& \quad \leq \frac{f\left(\frac{1}{a}\right)+f\left(\frac{1}{b}\right)}{2}\left({ }_{g} \Upsilon_{\sigma, \tau, \delta, \omega^{\prime},\left(\frac{1}{b}\right)^{+}}^{\rho, r, k, c} 1\right)\left(\frac{1}{a} ; p\right),
\end{aligned}
$$

where $g$ is the reciprocal function. 
The following lemma is useful to give the next result.

Lemma 2.4 Let $f, g:[a, b] \rightarrow \mathbb{R}, 0<a<b$, Range $(g) \subset[a, b]$, be functions such that $f$ is positive, $f \in L_{1}[a, b]$, and $g$ is differentiable and strictly increasing. If $f\left(\frac{1}{g(x)}\right)=f\left(\frac{1}{\frac{1}{g(a)}+\frac{1}{g(b)}-g(x)}\right)$, then for operators (1.9) and (1.10) we have:

$$
\begin{aligned}
& \left({ }_{g} \Upsilon_{\sigma, \tau, \delta, \omega,\left(g^{-1}\left(\frac{1}{g(b)}\right)\right)+}^{\rho, r, k} f \circ \psi\right)\left(g^{-1}\left(\frac{1}{g(a)}\right) ; p\right) \\
& =\left({ }_{g} \Upsilon_{\sigma, \tau, \delta, \omega,\left(g^{-1}\left(\frac{1}{g(a)}\right)\right)}^{\rho, r, k} f \circ \psi\right)\left(g^{-1}\left(\frac{1}{g(b)}\right) ; p\right) \\
& =\frac{1}{2}\left(\left({ }_{g} \Upsilon_{\sigma, \tau, \delta, \omega,\left(g^{-1}\left(\frac{1}{g(b)}\right)\right)}^{\rho, r, k}, f \circ \psi\right)\left(g^{-1}\left(\frac{1}{g(a)}\right) ; p\right)\right. \\
& \left.+\left({ }_{g} \Upsilon_{\sigma, \tau, \delta, \omega,\left(g^{-1}\left(\frac{1}{g(a)}\right)\right)}^{\rho, r, k} f \circ \psi\right)\left(g^{-1}\left(\frac{1}{g(b)}\right) ; p\right)\right),
\end{aligned}
$$

where $\psi(t)=\frac{1}{g(t)}$ for all $t \in\left[\frac{1}{b}, \frac{1}{a}\right]$

Proof For operators (1.9) and (1.10), we can write

$$
\begin{aligned}
& \left({ }_{g} \Upsilon_{\sigma, \tau, \delta, \omega,\left(g^{-1}\left(\frac{1}{g(b)}\right)\right)}^{\rho, r, k, c} f \circ \psi\right)\left(g^{-1}\left(\frac{1}{g(a)}\right) ; p\right) \\
& \quad=\int_{g^{-1}\left(\frac{1}{g(b)}\right)}^{g^{-1}\left(\frac{1}{g(a)}\right)}\left(\frac{1}{g(a)}-g(t)\right)^{\tau-1} E_{\sigma, \tau, \delta}^{\rho, r, k, c}\left(\omega\left(\frac{1}{g(a)}-g(t)\right)^{\sigma} ; p\right) f\left(\frac{1}{g(t)}\right) d(g(t)) .
\end{aligned}
$$

Replacing $g(t)$ by $\frac{1}{g(a)}+\frac{1}{g(b)}-g(x)$ in equation (2.8) and then using $f\left(\frac{1}{g(x)}\right)=f\left(\frac{1}{\frac{1}{g(a)}+\frac{1}{g(b)}-g(x)}\right)$, we have

$$
\begin{aligned}
& \left({ }_{g} \Upsilon_{\sigma, \tau, \delta, \omega,\left(g^{-1}\left(\frac{1}{g(b)}\right)\right)+}^{\rho, r, k} f \circ \psi\right)\left(g^{-1}\left(\frac{1}{g(a)}\right) ; p\right) \\
& \quad=\left({ }_{g} \Upsilon_{\sigma, \tau, \delta,, \omega,\left(g^{-1}\left(\frac{1}{g(a)}\right)\right)}^{\rho, r, c} f \circ \psi\right)\left(g^{-1}\left(\frac{1}{g(b)}\right) ; p\right) .
\end{aligned}
$$

By adding $\left(g \Upsilon_{\sigma, \tau, \delta, \omega,\left(g^{-1}\left(\frac{1}{g(b)}\right)\right)+}^{\rho, r, k} f \circ \psi\right)\left(g^{-1}\left(\frac{1}{g(a)}\right) ; p\right)$ on both sides of (2.9), we have

$$
\begin{aligned}
2\left({ }_{g} \Upsilon_{\sigma, \tau, \delta, \omega,\left(g^{-1}\left(\frac{1}{g(b)}\right)\right)+}^{\rho, r, k} f \circ \psi\right)\left(g^{-1}\left(\frac{1}{g(a)}\right) ; p\right) \\
=\left({ }_{g} \Upsilon_{\sigma, \tau, \delta, \omega,\left(g^{-1}\left(\frac{1}{g(b)}\right)\right)+}^{\rho, r, k} f \circ \psi\right)\left(g^{-1}\left(\frac{1}{g(a)}\right) ; p\right) \\
\quad+\left({ }_{g} \Upsilon_{\sigma, \tau, \delta, \omega, c,\left(g^{-1}\left(\frac{1}{g(a)}\right)\right)}^{\rho, r, c} f \circ \psi\right)\left(g^{-1}\left(\frac{1}{g(b)}\right) ; p\right) .
\end{aligned}
$$

Equations (2.9) and (2.11) give required result.

Theorem 2.5 Let $f, g, h:[a, b] \rightarrow \mathbb{R}, 0<a<b$, Range $(g)$, Range $(h) \subset[a, b]$, be functions such that $f$ is positive, $f \in L_{1}[a, b], g$ is differentiable, strictly increasing, and $h$ is nonnegative and integrable. If $f$ is harmonically convex and $f\left(\frac{1}{g(x)}\right)=f\left(\frac{1}{\frac{1}{g(a)}+\frac{1}{g(b)}-g(x)}\right)$, then for 
fractional integral operators (1.9) and (1.10) we have:

$$
\begin{aligned}
& f\left(\frac{2 g(a) g(b)}{g(a)+g(b)}\right)\left(\left({ }_{g} \Upsilon_{\sigma, \tau, \delta, \omega^{\prime},\left(g^{-1}\left(\frac{1}{g(b)}\right)\right)^{+}}^{\rho, r, k} h \circ \psi\right)\left(g^{-1}\left(\frac{1}{g(a)}\right) ; p\right)\right. \\
& \left.+\left({ }_{g} \Upsilon_{\sigma, \tau, \delta, \omega^{\prime},\left(g^{-1}\left(\frac{1}{g(a)}\right)\right)^{\rho, r, k}} h \circ \psi\right)\left(g^{-1}\left(\frac{1}{g(b)}\right) ; p\right)\right) \\
& \leq\left({ }_{g} \Upsilon_{\sigma, \tau, \delta, \omega^{\prime},\left(g^{-1}\left(\frac{1}{g(b)}\right)\right)}^{\rho, r, k} f h \circ \psi\right)\left(g^{-1}\left(\frac{1}{g(a)}\right) ; p\right) \\
& +\left({ }_{g} \Upsilon_{\sigma, \tau, \delta, \omega^{\prime},\left(g^{-1}\left(\frac{1}{g(a)}\right)\right)}^{\rho, r, k} f h \circ \psi\right)\left(g^{-1}\left(\frac{1}{g(b)}\right) ; p\right) \\
& \leq \frac{f(g(a))+f(g(b))}{2}\left(\left({ }_{g} \Upsilon_{\sigma, \tau, \delta, \omega^{\prime},\left(g^{-1}\left(\frac{1}{g(b)}\right)\right)^{+}}^{\rho, r, k} h \psi \psi\right)\left(g^{-1}\left(\frac{1}{g(a)}\right) ; p\right)\right. \\
& \left.+\left({ }_{g} \Upsilon_{\sigma, \tau, \delta, \omega^{\prime},\left(g^{-1}\left(\frac{1}{g(a)}\right)\right)^{\rho, r, k, c}}^{\rho+} h \circ \psi\right)\left(g^{-1}\left(\frac{1}{g(b)}\right) ; p\right)\right),
\end{aligned}
$$

where $\psi(t)=\frac{1}{g(t)}$ for all $t \in\left[\frac{1}{b}, \frac{1}{a}\right]$, fho $\psi=(f \circ \psi)(h \circ \psi)$ and $\omega^{\prime}=\omega\left(\frac{g(a) g(b)}{g(b)-g(a)}\right)^{\sigma}$.

Proof Multiplying (2.3) by $t^{\tau-1} E_{\sigma, \tau, \delta}^{\rho, r, k, c}\left(\omega t^{\sigma} ; p\right) h\left(\frac{g(a) g(b)}{\operatorname{tg}(b)+(1-t) g(a)}\right)$, then integrating over [0,1] we get

$$
\begin{aligned}
2 f( & \left.\frac{2 g(a) g(b)}{g(a)+g(b)}\right) \int_{0}^{1} t^{\tau-1} E_{\sigma, \tau, \delta}^{\rho, r, k, c}\left(\omega t^{\sigma} ; p\right) h\left(\frac{g(a) g(b)}{\operatorname{tg}(b)+(1-t) g(a)}\right) d t \\
\leq & \int_{0}^{1} t^{\tau-1} E_{\sigma, \tau, \delta}^{\rho, r, k, c}\left(\omega t^{\sigma} ; p\right) f\left(\frac{g(a) g(b)}{\operatorname{tg}(a)+(1-t) g(b)}\right) h\left(\frac{g(a) g(b)}{\operatorname{tg}(b)+(1-t) g(a)}\right) d t \\
& +\int_{0}^{1} t^{\tau-1} E_{\sigma, \tau, \delta}^{\rho, r, k, c}\left(\omega t^{\sigma} ; p\right) f\left(\frac{g(a) g(b)}{\operatorname{tg}(b)+(1-t) g(a)}\right) h\left(\frac{g(a) g(b)}{\operatorname{tg}(b)+(1-t) g(a)}\right) d t .
\end{aligned}
$$

By setting $g(x)=\frac{\operatorname{tg}(b)+(1-t) g(a)}{g(a) g(b)}$ in (2.13) and using (1.9), (1.10), as well as the condition $f\left(\frac{1}{g(x)}\right)=f\left(\frac{1}{\frac{1}{g(a)}+\frac{1}{g(b)}-g(x)}\right)$, we have

$$
\begin{aligned}
2 f( & \left.\frac{2 g(a) g(b)}{g(a)+g(b)}\right)\left({ }_{g} \Upsilon_{\sigma, \tau, \delta, \omega^{\prime},\left(g^{-1}\left(\frac{1}{g(a)}\right)\right)^{\rho}}^{\rho, r, k, c} h \circ \psi\right)\left(g^{-1}\left(\frac{1}{g(b)}\right) ; p\right) \\
\leq & \left({ }_{g} \Upsilon_{\sigma, \tau, \delta, \omega^{\prime},\left(g^{-1}\left(\frac{1}{g(b)}\right)\right)^{\rho+}}^{\rho, r, k} f h \circ \psi\right)\left(g^{-1}\left(\frac{1}{g(a)}\right) ; p\right) \\
& +\left({ }_{g} \Upsilon_{\sigma, \tau, \delta, \omega^{\prime},\left(g^{-1}\left(\frac{1}{g(a)}\right)\right)}^{\rho, r, k, c} f h \circ \psi\right)\left(g^{-1}\left(\frac{1}{g(b)}\right) ; p\right) .
\end{aligned}
$$

By using Lemma 2.4 in the above inequality, one can get the first inequality of (2.12). For the second inequality of (2.12), multiplying $(2.5)$ by $t^{\tau-1} E_{\sigma, \tau, \delta}^{\rho, r, k, c}\left(\omega t^{\sigma} ; p\right) h\left(\frac{g(a) g(b)}{\operatorname{tg}(b)+(1-t) g(a)}\right)$, then integrating over $[0,1]$, we get

$$
\begin{aligned}
& \int_{0}^{1} t^{\tau-1} E_{\sigma, \tau, \delta}^{\rho, r, k}\left(\omega t^{\sigma} ; p\right) f\left(\frac{g(a) g(b)}{\operatorname{tg}(b)+(1-t) g(a)}\right) h\left(\frac{g(a) g(b)}{\operatorname{tg}(b)+(1-t) g(a)}\right) d t \\
& \quad+\int_{0}^{1} t^{\tau-1} E_{\sigma, \tau, \delta}^{\rho, r, k, c}\left(\omega t^{\sigma} ; p\right) f\left(\frac{g(a) g(b)}{\operatorname{tg}(a)+(1-t) g(b)}\right) h\left(\frac{g(a) g(b)}{\operatorname{tg}(b)+(1-t) g(a)}\right) d t
\end{aligned}
$$




$$
\leq(f(g(a))+f(g(b))) \int_{0}^{1} t^{\tau-1} E_{\sigma, \tau, \delta}^{\rho, r, k, c}\left(\omega t^{\sigma} ; p\right) h\left(\frac{g(a) g(b)}{\operatorname{tg}(b)+(1-t) g(a)}\right) d t .
$$

Setting $g(x)=\frac{\operatorname{tg}(b)+(1-t) g(a)}{g(a) g(b)}$ in (2.15) and using (1.9), (1.10), as well as the condition $f\left(\frac{1}{g(x)}\right)=$ $f\left(\frac{1}{\frac{1}{g(a)}+\frac{1}{g(b)}-g(x)}\right)$, we have

$$
\begin{aligned}
& \left({ }_{g} \Upsilon_{\sigma, \tau, \delta, \omega^{\prime},\left(g^{-1}\left(\frac{1}{g(b)}\right)\right)^{+}}^{\rho, r, k} f h \circ \psi\right)\left(g^{-1}\left(\frac{1}{g(a)}\right) ; p\right) \\
& \quad+\left(g \Upsilon_{\sigma, \tau, \delta, \omega^{\prime},\left(g^{-1}\left(\frac{1}{g(a)}\right)\right)}^{\rho, r, k} f h \circ \psi\right)\left(g^{-1}\left(\frac{1}{g(b)}\right) ; p\right) \\
& \leq(f(g(a))+f(g(b)))\left({ }_{g} \Upsilon_{\sigma, \tau, \delta, \omega^{\prime},\left(g^{-1}\left(\frac{1}{g(b)}\right)\right)^{+}}^{\rho, r, k} \circ \psi\right)\left(g^{-1}\left(\frac{1}{g(a)}\right) ; p\right) .
\end{aligned}
$$

Again using Lemma 2.4 in (2.16), one can get the second inequality of (2.12).

\section{Remark 2.6}

(i) By setting $p=0, h(x)=1$ and $g=I$, [1, Theorem 3.1] is obtained.

(ii) By setting $g=I$ and $h(x)=1$, [9, Theorem 2.1] is obtained.

(iii) By setting $\omega=p=0, h(x)=1$ and $g=I$, [15, Theorem 4] is obtained.

(iv) By setting $\omega=p=0, \tau=1$ and $g=I$, [3, Theorem 8] is obtained.

(v) By setting $\omega=p=0, \tau=1, h(x)=1$ and $g=I$, [25, Theorem 2.4] is obtained.

Theorem 2.7 Let $f, g:[a, b] \rightarrow \mathbb{R}, 0<a<b$, Range $(g) \subset[a, b]$, be functions such that $f$ is positive, $f \in L_{1}[a, b]$, and $g$ is differentiable and strictly increasing. Iff is harmonically convex on $[a, b]$ and $f\left(\frac{1}{g(x)}\right)=f\left(\frac{1}{\frac{1}{g(a)}+\frac{1}{g(b)}-g(x)}\right)$, then for operators (1.9) and (1.10) we have:

$$
\begin{aligned}
& f\left(\frac{2 g(a) g(b)}{g(a)+g(b)}\right)\left({ }_{g} \Upsilon_{\sigma, \tau, \delta, \omega^{\prime},\left(g^{-1}\left(\frac{g(a)+g(b)}{2 g(a) g(b)}\right)\right)}^{\rho, r, k}-1\right)\left(g^{-1}\left(\frac{1}{g(b)}\right) ; p\right) \\
& \leq \frac{1}{2}\left(\left(g_{\sigma, \tau, \delta, \omega^{\prime},\left(g^{-1}\left(\frac{g(a)+g(b)}{2 g(a) g(b)}\right)\right)}^{\rho, r, k, c} f \circ \psi\right)\left(g^{-1}\left(\frac{1}{g(a)}\right) ; p\right)\right. \\
& \left.+\left({ }_{g} \Upsilon_{\sigma, \tau, \delta, \omega^{\prime},\left(g^{-1}\left(\frac{g(a)+g(b)}{2 g(a) g(b)}\right)\right)}^{\rho, r, k,}-f \circ \psi\right)\left(g^{-1}\left(\frac{1}{g(b)}\right) ; p\right)\right) \\
& \leq \frac{f(g(a))+f(g(b))}{2}\left({ }_{g} \Upsilon_{\sigma, \tau, \delta, \omega^{\prime},\left(g^{-1}\left(\frac{g(a)+g(b)}{2 g(a) g(b)}\right)\right)}^{\rho, r, k,}+1\right)\left(g^{-1}\left(\frac{1}{g(a)}\right) ; p\right) \text {, }
\end{aligned}
$$

where $\psi(t)=\frac{1}{g(t)}$ for $t \in\left[\frac{1}{b}, \frac{1}{a}\right]$ and $\omega^{\prime}=\omega\left(\frac{g(a) g(b)}{g(b)-g(a)}\right)^{\sigma}$.

Proof Multiplying (2.3) by $2 t^{\tau-1} E_{\sigma, \tau, \delta}^{\rho, r, k, c}\left(\omega t^{\sigma} ; p\right)$ then integrating over $\left[0, \frac{1}{2}\right]$, we have

$$
\begin{aligned}
2 f( & \left.\frac{2 g(a) g(b)}{g(a)+g(b)}\right) \int_{0}^{\frac{1}{2}} t^{\tau-1} E_{\sigma, \tau, \delta}^{\rho, r, k, c}\left(\omega t^{\sigma} ; p\right) d t \\
\leq & \int_{0}^{\frac{1}{2}} t^{\tau-1} E_{\sigma, \tau, \delta}^{\rho, r, k, c}\left(\omega t^{\sigma} ; p\right) f\left(\frac{g(a) g(b)}{\operatorname{tg}(a)+(1-t) g(b)}\right) d t \\
& \quad+\int_{0}^{\frac{1}{2}} t^{\tau-1} E_{\sigma, \tau, \delta}^{\rho, r, k, c}\left(\omega t^{\sigma} ; p\right) f\left(\frac{g(a) g(b)}{\operatorname{tg}(b)+(1-t) g(a)}\right) d t .
\end{aligned}
$$


Setting $g(x)=\frac{\operatorname{tg}(b)+(1-t) g(a)}{g(a) g(b)}$ in $(2.18)$ and using $f\left(\frac{1}{g(x)}\right)=f\left(\frac{1}{\frac{1}{g(a)}+\frac{1}{g(b)}-g(x)}\right)$, as well as (1.9) and (1.10), the first inequality of (2.17) can be obtained.

For the second inequality, multiplying (2.5) by $t^{\tau-1} E_{\sigma, \tau, \delta}^{\rho, r, k, c}\left(\omega t^{\sigma} ; p\right)$ then integrating over $\left[0, \frac{1}{2}\right]$, we get

$$
\begin{gathered}
\int_{0}^{\frac{1}{2}} t^{\tau-1} E_{\sigma, \tau, \delta}^{\rho, r, k, c}\left(\omega t^{\sigma} ; p\right) f\left(\frac{g(a) g(b)}{\operatorname{tg}(b)+(1-t) g(a)}\right) d t \\
\quad+\int_{0}^{\frac{1}{2}} t^{\tau-1} E_{\sigma, \tau, \delta}^{\rho, r, k, c}\left(\omega t^{\sigma} ; p\right) f\left(\frac{g(a) g(b)}{\operatorname{tg}(a)+(1-t) g(b)}\right) d t \\
\leq(f(g(a))+f(g(b))) \int_{0}^{\frac{1}{2}} t^{\tau-1} E_{\sigma, \tau, \delta}^{\rho, r, k, c}\left(\omega t^{\sigma} ; p\right) d t .
\end{gathered}
$$

Setting $g(x)=\frac{\operatorname{tg}(b)+(1-t) g(a)}{g(a) g(b)}$ in (2.19) and using $f\left(\frac{1}{g(x)}\right)=f\left(\frac{1}{\frac{1}{g(a)}+\frac{1}{g(b)}-g(x)}\right)$, as well as (1.9) and (1.10), the second inequality of (2.17) can be obtained.

\section{Remark 2.8}

(i) By setting $p=0$ and $g=I$, [1, Theorem 3.3] is obtained.

(ii) By setting $g=I$, [9, Theorem 2.3] is obtained.

(iii) By setting $\omega=p=0$ and $g=I,[25$, Theorem 4$]$ is obtained.

To prove the next result, we will use the following lemma:

Lemma 2.9 Let $f, g:[a, b] \rightarrow \mathbb{R}, 0<a<b$, Range $(g) \subset[a, b]$, be functions such that $f$ is positive, $f \in L_{1}[a, b]$, and $g$ is differentiable and strictly increasing. If $f$ is harmonically convex and $f\left(\frac{1}{g(x)}\right)=f\left(\frac{1}{\frac{1}{g(a)}+\frac{1}{g(b)}-g(x)}\right)$, then for fractional integral operators (1.9) and (1.10) we have:

$$
\begin{aligned}
& \left({ }_{g} \Upsilon_{\sigma, \tau, \delta, \omega,\left(g^{-1}\left(\frac{g(a)+g(b)}{2 g(a) g(b)}\right)\right)}^{\rho, r, k, c} f \circ \psi\right)\left(g^{-1}\left(\frac{1}{g(a)}\right) ; p\right) \\
& =\left({ }_{g} \Upsilon_{\sigma, \tau, \delta, \omega,\left(g^{-1}\left(\frac{g(a)+g(b)}{2 g(a) g(b)}\right)\right)}^{\rho, r, k, c} f \circ \psi\right)\left(g^{-1}\left(\frac{1}{g(b)}\right) ; p\right) \\
& =\frac{1}{2}\left(\left({ }_{g} \Upsilon_{\sigma, \tau, \delta, \omega,\left(g^{-1}\left(\frac{g(a)+g(b)}{2 g(a) g(b)}\right)\right)}^{\rho, r, k, c} f \circ \psi\right)\left(g^{-1}\left(\frac{1}{g(a)}\right) ; p\right)\right. \\
& \left.\quad+\left({ }_{g} \Upsilon_{\sigma, \tau, \delta, \omega,\left(g^{-1}\left(\frac{g(a)+g(b)}{2 g(a) g(b)}\right)\right)}^{\rho, r, k, c} f \circ \psi\right)\left(g^{-1}\left(\frac{1}{g(b)}\right) ; p\right)\right), \\
& \psi(t)=\frac{1}{g(t)}, t \in\left[\frac{1}{b}, \frac{1}{a}\right] .
\end{aligned}
$$

Proof By using Definition 4, we can write

$$
\begin{aligned}
& \left({ }_{g} \Upsilon_{\sigma, \tau, \delta, \omega,\left(g^{-1}\left(\frac{g(a)+g(b)}{2 g(a) g(b)}\right)\right)^{+}}^{\rho, r, k, c} f \circ \psi\right)\left(g^{-1}\left(\frac{1}{g(a)}\right) ; p\right) \\
& \quad=\int_{g^{-1}\left(\frac{g(a)+g(b)}{2 g(a) g(b)}\right)}^{g^{-1}\left(\frac{1}{g(a)}\right)}\left(\frac{1}{g(a)}-g(t)\right)^{\tau-1} E_{\sigma, \tau, \delta}^{\rho, r, k, c}\left(\omega\left(\frac{1}{g(a)}-g(t)\right)^{\sigma} ; p\right) f\left(\frac{1}{g(t)}\right) d(g(t)) .
\end{aligned}
$$


By replacing $g(t)$ with $\frac{1}{g(a)}+\frac{1}{g(b)}-g(x)$ in equation (2.21) and using the condition $f\left(\frac{1}{g(x)}\right)=$ $f\left(\frac{1}{\frac{1}{g(a)}+\frac{1}{g(b)}-g(x)}\right)$, we have

$$
\begin{aligned}
& \left({ }_{g} \Upsilon_{\sigma, \tau, \delta, \omega,\left(g^{-1}\left(\frac{g(a)+g(b)}{2 g(a) g(b)}\right)\right)+}^{\rho, r, k, c} f \circ \psi\right)\left(g^{-1}\left(\frac{1}{g(a)}\right) ; p\right) \\
& \quad=\left({ }_{g} \Upsilon_{\sigma, \tau, \delta, \omega,\left(g^{-1}\left(\frac{g(a)+g(b)}{2 g(a) g(b)}\right)\right)}^{\rho, r, k, c} f \circ \psi\right)\left(g^{-1}\left(\frac{1}{g(b)}\right) ; p\right) .
\end{aligned}
$$

By adding $\left({ }_{g} \Upsilon_{\sigma, \tau, \delta, \omega,\left(g^{-1}\left(\frac{g(a)+g(b)}{2 g(a) g(b)}\right)\right)^{+}}^{\rho, r, k, c} f \circ \psi\right)\left(g^{-1}\left(\frac{1}{g(a)}\right) ; p\right)$ on both sides of (2.22), we have

$$
\begin{aligned}
& 2\left({ }_{g} \Upsilon_{\sigma, \tau, \delta, \omega,\left(g^{-1}\left(\frac{g(a)+g(b)}{2 g(a) g(b)}\right)\right)^{+}}^{\rho, r, k, c} f \circ \psi\right)\left(g^{-1}\left(\frac{1}{g(a)}\right) ; p\right) \\
& =\left({ }_{g} \Upsilon_{\sigma, \tau, \delta, \omega,\left(g^{-1}\left(\frac{g(a)+g(b)}{2 g(a) g(b)}\right)\right)}^{\rho, r, k, c} f \circ \psi\right)\left(g^{-1}\left(\frac{1}{g(a)}\right) ; p\right) \\
& \quad+\left({ }_{g} \Upsilon_{\sigma, \tau, \delta, \omega,\left(g^{-1}\left(\frac{g(a)+g(b)}{2 g(a) g(b)}\right)\right)}^{\rho, r, k, c} f \circ \psi\right)\left(g^{-1}\left(\frac{1}{g(b)}\right) ; p\right)
\end{aligned}
$$

Equations (2.22) and (2.23) give the required result.

Theorem 2.10 Let $f, g, h:[a, b] \rightarrow \mathbb{R}, 0<a<b$, Range $(g)$, Range $(h) \subset[a, b]$, be functions such that $f$ is positive, $f \in L_{1}[a, b], g$ is differentiable, strictly increasing, and $h$ is nonnegative and integrable. If $f$ is harmonically convex and $f\left(\frac{1}{g(x)}\right)=f\left(\frac{1}{\frac{1}{g(a)}+\frac{1}{g(b)}-g(x)}\right)$, then for fractional integral operators (1.9) and (1.10) we have

$$
\begin{aligned}
f( & \left.\frac{2 g(a) g(b)}{g(a)+g(b)}\right)\left(\left({ }_{g} \Upsilon_{\sigma, \tau, \delta, \omega^{\prime},\left(g^{-1}\left(\frac{g(a)+g(b)}{2 g(a) g(b)}\right)\right)^{+}}^{\rho, r, k, c} h \circ \psi\right)\left(g^{-1}\left(\frac{1}{g(a)}\right) ; p\right)\right. \\
& \left.+\left({ }_{g} \Upsilon_{\sigma, \tau, \delta, \omega^{\prime},\left(g^{-1}\left(\frac{g(a)+g(b)}{2 g(a) g(b)}\right)\right)^{-}}^{\rho, r, k, c} h \circ \psi\right)\left(g^{-1}\left(\frac{1}{g(b)}\right) ; p\right)\right) \\
\leq & \left({ }_{g} \Upsilon_{\sigma, \tau, \delta, \omega^{\prime},\left(g^{-1}\left(\frac{g(a)+g(b)}{2 g(a) g(b)}\right)\right)^{+}}^{\rho, r, k, c} f h \circ \psi\right)\left(g^{-1}\left(\frac{1}{g(a)}\right) ; p\right) \\
& +\left({ }_{g} \Upsilon_{\sigma, \tau, \delta, \omega^{\prime},\left(g^{-1}\left(\frac{g(a)+g(b)}{2 g(a) g(b)}\right)\right)^{\rho, r, k}, c} f h \circ \psi\right)\left(g^{-1}\left(\frac{1}{g(b)}\right) ; p\right) \\
\leq & \frac{f(g(a))+f(g(b))}{2}\left(\left({ }_{g} \Upsilon_{\sigma, \tau, \delta, \omega^{\prime},\left(g^{-1}\left(\frac{g(a)+g(b)}{2 g(a) g(b)}\right)\right)^{+}}^{\rho, r, k, c} \psi \psi\right.\right. \\
& \left.+\left(g_{g} \Upsilon_{\sigma, \tau, \delta, \omega^{\prime},\left(g^{-1}\left(\frac{g(a)+g(b)}{2 g(a) g(b)}\right)\right)^{-}}^{\rho, r, k, c} h \circ \psi\right)\left(g^{-1}\left(\frac{1}{g(b)}\right) ; p\right)\right),
\end{aligned}
$$

where $\psi(t)=\frac{1}{g(t)}$ for $t \in\left[\frac{1}{b}, \frac{1}{a}\right]$, fh $\circ \psi=(f \circ \psi)(h \circ \psi)$ and $\omega^{\prime}=\omega\left(\frac{g(a) g(b)}{g(b)-g(a)}\right)^{\sigma}$.

Proof Multiplying (2.3) by $t^{\tau-1} E_{\sigma, \tau, \delta}^{\rho, r, k, c}\left(\omega t^{\sigma} ; p\right) h\left(\frac{g(a) g(b)}{\operatorname{tg}(b)+(1-t) g(a)}\right)$ then integrating over $\left[0, \frac{1}{2}\right]$, we have

$$
2 f\left(\frac{2 g(a) g(b)}{g(a)+g(b)}\right) \int_{0}^{\frac{1}{2}} t^{\tau-1} E_{\sigma, \tau, \delta}^{\rho, r, k, c}\left(\omega t^{\sigma} ; p\right) h\left(\frac{g(a) g(b)}{\operatorname{tg}(b)+(1-t) g(a)}\right) d t
$$




$$
\begin{aligned}
\leq & \int_{0}^{\frac{1}{2}} t^{\tau-1} E_{\sigma, \tau, \delta}^{\rho, r, k, c}\left(\omega t^{\sigma} ; p\right) f\left(\frac{g(a) g(b)}{\operatorname{tg}(a)+(1-t) g(b)}\right) h\left(\frac{g(a) g(b)}{\operatorname{tg}(b)+(1-t) g(a)}\right) d t \\
& +\int_{0}^{\frac{1}{2}} t^{\tau-1} E_{\sigma, \tau, \delta}^{\rho, r, k, c}\left(\omega t^{\sigma} ; p\right) f\left(\frac{g(a) g(b)}{\operatorname{tg}(b)+(1-t) g(a)}\right) h\left(\frac{g(a) g(b)}{\operatorname{tg}(b)+(1-t) g(a)}\right) d t .
\end{aligned}
$$

By choosing $g(x)=\frac{\operatorname{tg}(b)+(1-t) g(a)}{g(a) g(b)}$ and using the condition $f\left(\frac{1}{g(x)}\right)=f\left(\frac{1}{\frac{1}{g(a)}+\frac{1}{g(b)}-g(x)}\right)$ in (2.25), we have

$$
\begin{aligned}
2 f( & \left.\frac{2 g(a) g(b)}{g(a)+g(b)}\right)\left({ }_{g} \Upsilon_{\sigma, \tau, \delta, \omega^{\prime},\left(g^{-1}\left(\frac{g(a)+g(b)}{2 g(a) g(b)}\right)\right)^{-}}^{\rho, r k, c} h \circ \psi\right)\left(g^{-1}\left(\frac{1}{g(b)}\right) ; p\right) \\
\leq & \left({ }_{g} \Upsilon_{\sigma, \tau, \delta, \omega^{\prime},\left(g^{-1}\left(\frac{g(a)+g(b)}{2 g(a) g(b))}\right)\right)^{\rho}}^{\rho, r, k,} f h \circ \psi\right)\left(g^{-1}\left(\frac{1}{g(a)}\right) ; p\right) \\
& +\left({ }_{g} \Upsilon_{\sigma, \tau, \delta, \omega^{\prime},\left(g^{-1}\left(\frac{g(a)+g(b)}{2 g(a) g(b)}\right)\right)}^{\rho, r, k, c} f h \circ \psi\right)\left(g^{-1}\left(\frac{1}{g(b)}\right) ; p\right) .
\end{aligned}
$$

Using Lemma 2.9 in the above inequality, one can get the first inequality of (2.24). For the second part of inequality of (2.24), multiplying (2.5) by $t^{\tau-1} E_{\sigma, \tau, \delta}^{\rho, r, k, c}\left(\omega t^{\sigma} ; p\right) h\left(\frac{g(a) g(b)}{\operatorname{tg}(b)+(1-t) g(a)}\right)$ then integrating over $\left[0, \frac{1}{2}\right]$, we have

$$
\begin{aligned}
& \int_{0}^{\frac{1}{2}} t^{\tau-1} E_{\sigma, \tau, \delta}^{\rho, r, k, c}\left(\omega t^{\sigma} ; p\right) f\left(\frac{g(a) g(b)}{\operatorname{tg}(b)+(1-t) g(a)}\right) h\left(\frac{g(a) g(b)}{\operatorname{tg}(b)+(1-t) g(a)}\right) d t \\
& \quad+\int_{0}^{\frac{1}{2}} t^{\tau-1} E_{\sigma, \tau, \delta}^{\rho, r, k, c}\left(\omega t^{\sigma} ; p\right) f\left(\frac{g(a) g(b)}{\operatorname{tg}(a)+(1-t) g(b)}\right) h\left(\frac{g(a) g(b)}{\operatorname{tg}(b)+(1-t) g(a)}\right) d t \\
& \leq(f(g(a))+f(g(b))) \int_{0}^{\frac{1}{2}} t^{\tau-1} E_{\sigma, \tau, \delta}^{\rho, r, k, c}\left(\omega t^{\sigma} ; p\right) h\left(\frac{g(a) g(b)}{\operatorname{tg}(b)+(1-t) g(a)}\right) d t .
\end{aligned}
$$

Setting $g(x)=\frac{\operatorname{tg}(b)+(1-t) g(a)}{g(a) g(b)}$ in (2.27) and using (1.9), (1.10), as well as the condition $f\left(\frac{1}{g(x)}\right)=$ $f\left(\frac{1}{\frac{1}{g(a)}+\frac{1}{g(b)}-g(x)}\right)$, we have

$$
\begin{aligned}
& \left(g \Upsilon_{\sigma, \tau, \delta, \omega^{\prime},\left(g^{-1}\left(\frac{g(a)+g(b)}{2 g(a) g(b))}\right)+\right.}^{\rho, r, k} f h \circ \psi\right)\left(g^{-1}\left(\frac{1}{g(a)}\right) ; p\right) \\
& \quad+\left({ }_{g} \Upsilon_{\sigma, \tau, \delta, \omega^{\prime},\left(g ^ { - 1 } \left(\frac{g(a)+g(b)}{2 g(a) g(b)))}\right.\right.}^{\rho, r, k, c} f h \circ \psi\right)\left(g^{-1}\left(\frac{1}{g(b)}\right) ; p\right) \\
& \leq(f(g(a))+f(g(b)))\left({ }_{g} \Upsilon_{\sigma, \tau, \delta,, \omega^{\prime},\left(g^{-1}\left(\frac{g(a)+g(b)}{2 g(a) g(b)}\right)\right)^{+}}^{\rho, r, k}, h \circ \psi\right)\left(g^{-1}\left(\frac{1}{g(a)}\right) ; p\right) .
\end{aligned}
$$

Again using Lemma 2.9 in (2.28), the second inequality of (2.24) can be obtained.

\section{Remark 2.11}

(i) By setting $p=0$ and $g=I$, [1, Theorem 3.6] is obtained.

(ii) By setting $g=I$, [9, Theorem 2.6] is obtained.

(iii) By setting $\omega=p=0, g=I$ and $\tau=1$, [3, Theorem 8], is obtained. 
Corollary 2.12 Setting $\omega=p=0$ and $g=I$ in Theorem 2.10, we get the following inequalities via Riemann-Liouville fractional integrals:

$$
\begin{aligned}
& f\left(\frac{2 a b}{a+b}\right)\left(\left(I_{\frac{a+b}{2 a b}}^{\tau} h \circ \psi\right)\left(\frac{1}{a}\right)+\left(I_{\frac{a+b}{2 a b}}^{\tau} h \circ \psi\right)\left(\frac{1}{b}\right)\right) \\
& \quad \leq\left(I_{\frac{a+b}{2 a b}}^{\tau} f h \circ \psi\right)\left(\frac{1}{a}\right)+\left(I_{\frac{a+b}{2 a b}}^{\tau}-f h \circ \psi\right)\left(\frac{1}{b}\right) \\
& \quad \leq \frac{f(a)+f(b)}{2}\left(\left(I_{\frac{a+b}{2 a b}}^{\tau} h \circ \psi\right)\left(\frac{1}{a}\right)+\left(I_{\frac{a+b}{2 a b}}^{\tau}-h \circ \psi\right)\left(\frac{1}{b}\right)\right) .
\end{aligned}
$$

\section{Concluding remarks}

This paper investigates generalized fractional integral inequalities of Hadamard and Fejér-Hadamard-type for harmonically convex functions. Presented results are generalizations of several inequalities given in $[1,3,9,15,25]$. The results of this paper also hold for fractional integral operators defined in $[2,31,32,35,40]$ and are deducible from the generalized fractional integral operators given in (1.9) and (1.10), see [33, Remark 1].

\section{Acknowledgements}

We thank to the editor and referees for their careful reading and valuable suggestions to make the article reader-friendly. The research work of the second and fifth authors is supported by the Higher Education Commission of Pakistan with Project No. 5421 and Project No. 7962, respectively.

\section{Funding}

There is no funding available for the publication of this paper.

Availability of data and materials

There is no additional data required for the finding of results of this paper.

\section{Competing interests}

It is declared that authors have no competing interests.

\section{Authors' contributions}

All authors have equal contribution to this article. All authors read and approved the final manuscript.

\section{Author details}

${ }^{1}$ Institute of Computing Science and Technology, Guangzhou University, 510006, Guangzhou, China. ${ }^{2}$ Department of Mathematics, COMSATS University Islamabad, Attock Campus, Attock, Pakistan. ${ }^{3}$ Department of Mathematics, University of Sargodha, Sargodha, Pakistan.

\section{Publisher's Note}

Springer Nature remains neutral with regard to jurisdictional claims in published maps and institutional affiliations.

Received: 21 April 2020 Accepted: 8 July 2020 Published online: 17 July 2020

\section{References}

1. Abbas, G., Farid, G.: Hadamard and Fejér-Hadamard type inequalities for harmonically convex functions via generalized fractional integrals. J. Anal. 25(1), 107-119 (2017)

2. Andrić, M., Farid, G., Pečarić, J:: A generalization of Mittag-Leffler function associated with Opial type inequalities due to Mitrinović and Pečarić. Fract. Calc. Appl. Anal. 21(5), 1377-1395 (2018)

3. Chen, F., Wu, S.: Fejér and Hermite-Hadamard type inequalities for harmonically convex functions. J. Appl. Math. 2014, Article ID 386806 (2014)

4. Chen, H., Katugampola, U.N.: Hermite-Hadamard-Fejér type inequalities for generalized fractional integrals. J. Math. Anal. 446(2), 1274-1291 (2017)

5. Dahmani, Z:: On Minkowski and Hermite-Hadamard integral inequalities via fractional integration. Ann. Funct. Anal. $1(1), 51-58(2010)$

6. Ekinci, A., Ozdemir, M.E.: Some new integral inequalities via Riemann-Liouville integral operators. Appl. Comput. Math. 18(3), 288-295 (2019)

7. Farid, G.: A unified integral operator and further its consequences. Open J. Math. Anal. 4(1), 1-7 (2020)

8. Farid, G., Mishra, V.N., Mehmood, S.: Hadamard and the Fejér-Hadamard type inequalities for convex and relative convex function via an extended generalized Mittag-Leffler function. Int. J. Anal. Appl. 17(5), 892-903 (2019) 
9. Farid, G., Rehman, A.U., Mehmood, S.: Hadamard and Fejér-Hadamard type integral inequalities for harmonically convex functions via an extended generalized Mittag-Leffler function. J. Math. Comput. Sci. 8(5), 630-643 (2018)

10. Farid, G., Rehman, A.U., Zahra, M.: On Hadamard inequalities for relative convex functions via fractional integrals. Nonlinear Anal. Forum 21(1), 77-86 (2016)

11. Fejér, L.: Über die Fourierreihen II. Math. Naturwiss., Anz. Ungar. Akad. Wiss. 24, 369-390 (1906)

12. Giusti, A., Colombaro, I.: Probhakar-like fractional viscoelasticity. Commun. Nonlinear Sci. Numer. Simul. 56, 138-143 (2018)

13. Gorenflo, R., Kilbas, A.A., Mainardi, F., Rogosin, S.V.: Mittag-Leffler Functions, Related Topics and Applications. Springer, Berlin (2016)

14. Iscan, I.: Hermite-Hadamard type inequalities for harmonically convex functions. Hacet. J. Math. Stat. 43(6), 935-942 (2014)

15. Iscan, I., Wu, S.: Hermite-Hadamard type inequalities for harmonically convex functions via fractional integrals. Appl. Math. Comput. 238, 237-244 (2014)

16. Kang, S.M., Abbas, G., Farid, G., Nazeer, W.: A generalized Fejér-Hadamard inequality for harmonically convex functions via generalized fractional integral operator involving special functions and related results. Mathematics 6(7), $122(2018)$

17. Kang, S.M., Farid, G., Nazeer, W., Mehmood, S.: (h, $m$ )-Convex functions and associated fractional Hadamard and Fejer-Hadamard inequalities via an extended generalized Mittag-Leffler function. J. Inequal. Appl. 2019, 78 (2019)

18. Khan, A., Gomez-Aguilar, J.F., Khan, T.S., Khan, H.: Stability analysis and numerical solutions of fractional order HIV/AIDS model. Chaos Solitons Fractals 122, 119-128 (2019)

19. Khan, A., Khan, H., Gomez-Aguilar, J., Abdeljawad, T.: Existence and Hyers-Ulam stability for a nonlinear singular fractional differential equations with Mittag-Leffler kernel. Chaos Solitons Fractals 1(127), 422-427 (2019)

20. Khan, H., Jarad, F., Abdeljawad, T., Khan, A.: A singular ABC-fractional differential equation with p-Laplacian operator Chaos Solitons Fractals 1(129), 56-61 (2019)

21. Khan, H., Khan, A., Jarad, F., Shahd, A.: Existence and data dependence theorems for solutions of an ABC-fractional order impulsive system. Chaos Solitons Fractals 131, 109477 (2020). https://doi.org/10.1016/j.chaos.2019.109477

22. Khan, H., Li, Y., Khan, A., Khan, A.: Existence of solution for a fractional-order Lotka-Volterra reaction-diffusion model with Mittag-Leffler kernel. Math. Methods Appl. Sci. 42(9), 3377-3387 (2019). https://doi.org/10.1002/mma.5590

23. Khan, H., Tunc, C., Baleanu, D., Khan, A., Alkhazzan, A.: Inequalities for $n$-class of functions using the Saigo fractional integral operator. Rev. R. Acad. Cienc. Exactas Fís. Nat., Ser. A Mat. 113(3), 2407-2420 (2019)

24. Kilbas, A.A., Srivastava, H.M., Trujillo, J.J.: Theory and Applications of Fractional Differential Equations. North-Holland Mathematics Studies, vol. 204. Elsevier, New York (2006)

25. Kunt, M., Iscan, I., Yazi, N., Gozutok, U.: On new inequalities of Hermite-Hadamard-Fejér type inequalities for harmonically convex functions via fractional integrals. SpringerPlus 5(1), 1-19 (2016)

26. Kwun, Y.C., Farid, G., Ullah, S., Nazeer, W., Mahreen, K., Kang, S.M.: Inequalities for a unified integral operator and associated results in fractional calculus. IEEE Access 7, 126283-126292 (2019)

27. Mehmood, S., Farid, G., Khan, K.A., Yussouf, M.: New Hadamard and Fejér-Hadamard fractional inequalities for exponentially m-convex function. Eng. Appl. Sci. Lett. 3(1), 45-55 (2020)

28. Mittag-Leffler, G.M.: Sur la nouvelle fonction. C. R. Acad. Sci. Paris 137, 544-558 (1903)

29. Mubeen, S., Ali, R.S.: Fractional operators with generalized Mittag-Leffler k-function. Adv. Differ. Equ. 2019,520 (2019)

30. Mumcu, I., Set, E., Akdemir, A.O.: Hermite-Hadamard type inequalities for harmonically convex functions via Katugampola fractional integrals. Miskolc Math. Notes 20(1), 409-424 (2019)

31. Prabhakar, T.R.: A singular integral equation with a generalized Mittag-Leffler function in the kernel. Yokohama Math. J. 19, 7-15 (1971)

32. Rahman, G., Baleanu, D., Qurashi, M.A., Purohit, S.D., Mubeen, S., Arshad, M.: The extended generalized Mittag-Leffler function via fractional calculus. J. Nonlinear Sci. Appl. 10(1), 4244-4253 (2017)

33. Rao, Y., Yussouf, M., Farid, G., Pečarić, J., Tlili, I.: Further generalizations of Hadamard and Fejér-Hadamard inequalities and error estimations. Adv. Differ. Equ. (in press)

34. Rashid, S., Safdar, F., Akdemir, A.O., Noor, M.A., Noor, K.I.: Some new fractional integral inequalities for exponentially m-convex functions via extended generalized Mittag-Leffler function. J. Inequal. Appl. 2019, 299 (2019)

35. Salim, T.O., Faraj, A.W.: A generalization of Mittag-Leffler function and integral operator associated with integral calculus. J. Fract. Calc. Appl. 3(5), 1-13 (2012)

36. Sarikaya, M.Z., Alp, N.: On Hermite-Hadamard-Fejer type integral inequalities for generalized convex functions via local fractional integrals. Open J. Math. Sci. 3(1), 273-284 (2019)

37. Sarikaya, M.Z., Set, E., Yaldiz, H., Basak, N.: Hermite-Hadamard inequalities for fractional integrals and related fractional inequalities. Math. Comput. Model. 57, 2403-2407 (2013)

38. Sarikaya, M.Z., Yildirim, H.: On Hermite-Hadamard type inequalities for Riemann-Liouville fractional integrals. Miskolc Math. Notes 17(2), 1049-1059 (2017)

39. Shukla, A.K., Prajapati, J.C.: On a generalization of Mittag-Leffler function and its properties. J. Math. Anal. Appl. 336, 797-811 (2007)

40. Srivastava, H.M., Tomovski, Z:: Fractional calculus with an integral operator containing generalized Mittag-Leffler function in the kernel. Appl. Math. Comput. 211(1), 198-210 (2009)

41. Toader, G.H.: Some generalization of convexity. In: Proc. Colloq. Approx. Optim, Cluj Napoca (Romania), pp. 329-338 (1984)

42. Waheed, A., Farid, G., Rehman, A.U., Ayub, W.: k-Fractional integral inequalities for harmonically convex functions via Caputo k-fractional derivatives. Bull. Math. Anal. Appl. 10(1), 55-67 (2018)

43. Wiman, A.: Über den Fundamentalsatz in der Teorie der Funktionen $E_{a}(x)$. Acta Math. 29, 191-201 (1905)

44. Yaldiz, H., Akdemir, A.O.: Katugampola fractional integrals within the class of convex functions. Turk. J. Sci. III(I), 40-50 (2018) 